\title{
Structural Identification of Recombinant HEV VLP C-terminus
}

\author{
C.-Y. J. Wang, ${ }^{*}$ L. Xing, ${ }^{*}$ T.-C. Li, ${ }^{* *}$ N. Takeda, ${ }^{* *}$ Y. Yasutomi, ${ }^{* * *}$ D. J. Schofield,**** S. U. \\ Emerson, ${ }^{* * * *}$ R. H. Purcell, ${ }^{* * * *}$ T. Miyamura, ${ }^{* *}$ and R. H. Cheng*
}

* Section of molecular and cellular biology, University of California Davis, 1 Shields Avenue, Davis, CA 95616

** Department of Virology II, National Institute of Infectious Disease, Toyama 1-23-1, Shinjuku, Tokyo 162-8640, Japan

*** Department of Bioregulation, Mie University School of Medicine, Mie 514-8507, Japan

**** Hepatitis Viruses Section, Laboratory of Infectious Disease, National Institute of Allergy and Infectious Diseases, National Institute of Health, Bethesda, Maryland 20852

Background: Hepatitis E virus (HEV) is a fecal-oral transmitted epidemic disease that causes an acute liver failure in human. HEV holds a single stranded positive sensed RNA genome containing three open reading frames (ORFs), when expressing truncated ORF2 protein (PORF2) in the recombinant baculovirus system, the secreted proteins will self-assembled into virus-like particles (VLPs) [1-2]. We previously found that the deletion of 52 amino acids at PORF2 C-terminus is critical for forming the VLPs [3]; however, the detailed function and structural configuration of PORF2 C-terminus is still unknown. To address this question, in this study we took chimeric HEV VLP and antibodies as probes with the techniques of cryo-electron microscopy (cryo-EM) and threedimensional (3D) reconstruction to structurally identify the C-terminal location.

Results: The cryo-electron micrographs showed that VLP-Fab complex projected as spherical ringlike images decorated with spike-like features. The VLP-antibody complex was prepared by incubating the HEV capsid with the antibody Fab fragments of HEP224 (PORF2 epitope residues 601-608) and HEP4 (574-607) overnight. The 3D structure was determined according to the protocol using from icosahedral objects. The final reconstructions revealed that both antibodies interact with the exposing surface of PORF2 projection domain and the inserted 11 amino acids of chimeric HEV VLP locate at the top surface of protruding (P) domain. However, HEP224 gives better binding occupancy than that of HEP4, judged by relative height of the densities for VLP capsid and the bound Fabs. Our cryo-EM 3D structures have indicated the C-terminal route started from the lateral sides of two-fold P domain and oriented upward to the top surface. The footprints of two antibodies overlap at the top platform of PORF2 projection domain, suggesting the amino acids 601-608 located at the most outer surface of the viral particles. HEP4 shields more HEV VLP surface than HEP224, which correlates well to their linear epitope. The region that only involved in HEP4 footprint locates towards shell domain, thus the amino acids $574-600$ sit at stem region of PORF2 projecting spike. Combining the footprints of two antibodies together with chimeric HEV VLP, we could follow the route of C-terminal tail of truncated PORF2 from projecting stem to top platform. HEP4 has been demonstrated to be capable of neutralizing HEV challenge in experimental animals. Our finding of its epitope location could provide the structure basis for the study of its neutralizing mechanisms.

\section{References}

[1] T.C. Li et al., J. Virol. 71 (1997) 7207.
[2] L. Xing et al., Virology. 265 (1999) 35. 
[3] T.C. Li et al., J. Virol. 79 (2005) 12999.

[4] The research was sponsored by VR research council, STINT foundation, and a grant to Joseph C.-Y. Wang from National Science Council in Taiwan.
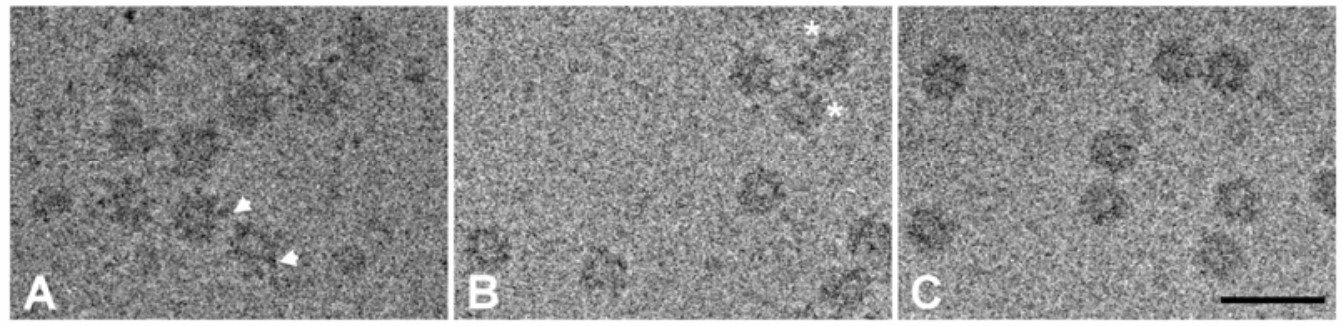

FIG. 1. Cryo-electron microscopy images of vitreous ice-embedded particles of VLP/HEV\#224 (A), VLP/HEV\#4 (B) and VLP/C-tag (C). The arrows in (A) denote the region of density attributed to HEV\#224 Fab fragments and the stars in (B) denotes the HEV\#4. The scale bar represents 50nm.

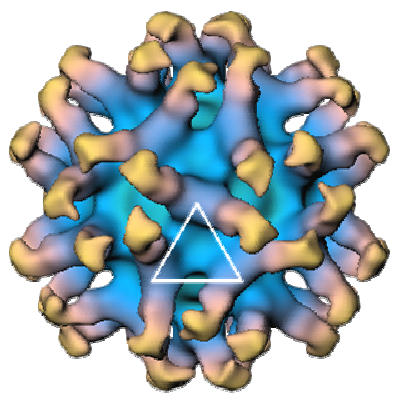

VLP/HEV\#224

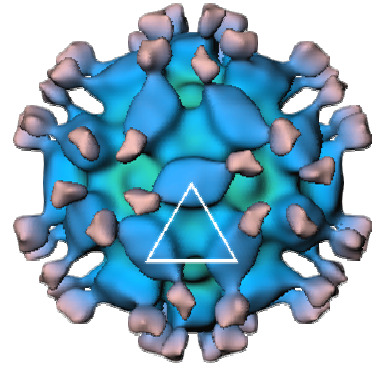

VLP/HEV\#4

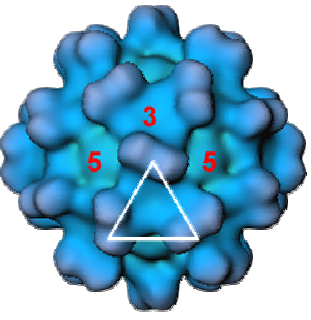

VLP/C-tag

0 10

$20(\mathrm{~nm})$

FIG. 2. Radial colored isosurface representations of VLP/HEV\#224, VLP/HEV\#4 and VLP/C-tag viewed along twofold axes. All the structures obey quasi-equivalent $T=1$ surface lattice and the three adjacent protruding spikes remain equal distance of $76 \AA$ (white lines) in the three structures. The scale bar represents $10 \mathrm{~nm}$.

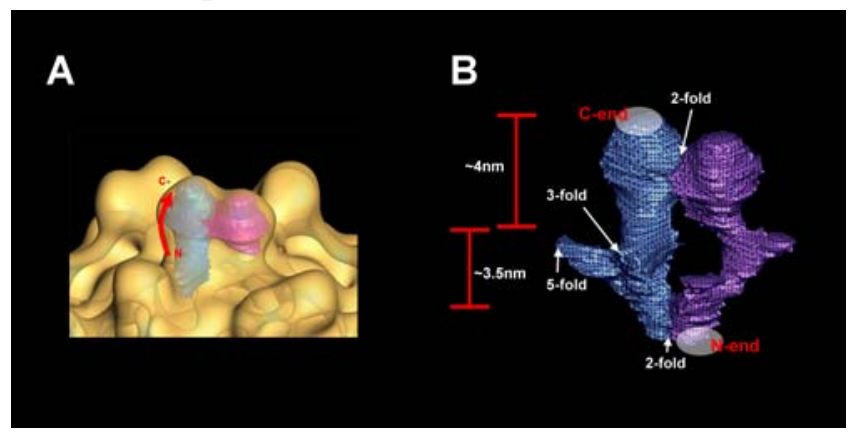

FIG. 3. The results indicated that the C-terminal tail locates at the lateral sides of $\mathrm{P}$ domain base, turns upward across the middle equatorial line, and ends at the top surface of the $\mathrm{P}$ domain. This region is also composed of the predominant antigenic site including the neutralizing epitope. 